\title{
Hypercalciuria in Postmenopausal Women With Reduced Bone Mineral Density Is Associated With Different Mineral Metabolic Profiles: Effects of Treatment With Thiazides and Anti-resorptives
}

\author{
Federico Nicoli ${ }^{1,2}$, Giorgia Dito ${ }^{2}$, Gregorio Guabello ${ }^{3}$, Matteo Longhi $^{3}$ and \\ Sabrina Corbetta ${ }^{2,4 *}$
}

${ }^{1}$ Department of Biotechnology and Translational Medicine, University of Milan, Milan, Italy, ${ }^{2}$ Endocrinology and Diabetology Service, IRCCS Istituto Ortopedico Galeazzi, Milan, Italy, ${ }^{3}$ Rheumatology Unit, IRCCS Istituto Ortopedico Galeazzi, Milan, Italy, ${ }^{4}$ Department of Biomedical, Surgical and Dental Sciences, University of Milan, Milan, Italy

\section{OPEN ACCESS}

Edited by:

Federica Saponaro,

University of Pisa, Italy

Reviewed by:

Antonino Catalano,

University of Messina, Italy

Jessica Pepe

Sapienza University of Rome, Italy

*Correspondence:

Sabrina Corbetta

sabrina.corbetta@unimi.it orcid.org/0000-0001-8140-3175

\section{Specialty section: \\ This article was submitted to \\ Pathology, \\ a section of the journa \\ Frontiers in Medicine}

Received: 20 September 2021 Accepted: 15 November 2021 Published: 15 December 2021

Citation:

Nicoli F, Dito G, Guabello G, Longhi M and Corbetta S (2021) Hypercalciuria in Postmenopausal Women With Reduced Bone Mineral Density Is Associated With Different Mineral Metabolic Profiles: Effects of

Treatment With Thiazides and Anti-resorptives.

Front. Med. 8:780087. doi: 10.3389/fmed.2021.780087
Hypercalciuria may represent a challenge during the workup for osteoporosis management. The present study aimed: (1) to describe the phenotype associated with hypercalciuria in vitamin D-sufficient (serum 25 hydroxyvitamin D $(25 \mathrm{OHD})>20 \mathrm{ng} / \mathrm{ml}$ ) patients with osteopenia/osteoporosis; (2) to analyze the effects of thiazides and antiresorptive drugs on urine calcium excretion (UCa), mineral metabolic markers, and bone mineral density. Seventy-seven postmenopausal women with hypercalciuria (Uca > 4.0 $\mathrm{mg} / \mathrm{kg}$ body weight $/ 24 \mathrm{~h}$ on two determinations) were retrospectively evaluated in a real-life setting. Median UCa was 5.39 (4.75-6.70) mg/kg/24 h. Kidney stones occurred in $32.9 \%$ of patients, who had median UCa similar to that of patients without kidney stones. Clustering analysis considering the three variables, such as serum calcium, phosphate, and parathormone (PTH), identified two main clusters of hypercalciuric patients. Cluster 1 ( $n=13$ ) included patients with a primary hyperparathyroidism-like profile, suggesting a certain degree of autonomous PTH secretion from parathyroid glands. Within cluster $2(n=61)$, two subgroups were recognized, cluster $2 \mathrm{~A}(n=18)$ that included patients with relatively increased PTH and normophosphatemia, and cluster 2B $(n=43)$ that included patients with the normal mineral profile. After a follow-up of $33.4 \pm 19.6$ months, 49 patients treated with thiazidic diuretics (TZD) were reevaluated; 20 patients were treated with hydrochlorothiazide (HCT; 12.5-37.5 mg/day), 29 with indapamide (IND; $1.50-3.75 \mathrm{mg} /$ day). Any significant difference could be detected in all the parameters both basal and treated conditions between patients treated with HCT or IND. TZD induced a mean 39\% reduction in UCa and $63.3 \%$ of patients obtained Uca $<4.0 \mathrm{mg} / \mathrm{kg} / 24 \mathrm{~h}$, independent of their mineral metabolic profile. Moreover, TZD induced a significant decrease in PTH levels. TZD-treated patients normalizing UCa experienced an increase in bone mineral densities when concomitantly treated with anti-resorptives, while any gain could be observed in TZD-treated patients with persistent hypercalciuria. Finally, multiple regression analysis showed that UCa reduction was at least in part 
related to denosumab treatment. In conclusion, in postmenopausal osteoporotic women, hypercalciuria is associated with kidney stones in about one-third of patients and with a wide range of impaired PTH secretion, determining a diagnostic challenge. TZD efficiently reduces $\mathrm{UCa}$ and normalization contributes to increasing anti-resorptives positive effect on bone mineral density.

Keywords: hypercalciuria, osteoporosis, PTH-parathyroid hormone, calcium, phosphate, hyperparathyroidism

\section{INTRODUCTION}

The role of hypercalciuria in kidney stones development has been extensively investigated in the general population (1) and patients with endocrine disorders, mainly in patients with primary hyperparathyroidism (2), while poor data are available about its role in bone metabolism derangement in the set of postmenopausal and aging osteoporosis. Hypercalciuria is frequently diagnosed during the workup for osteoporosis management (3), where it can be detected in up to onethird of patients with osteoporosis. Several metabolic disorders and medical treatments may promote hypercalciuria, such as primary hyperparathyroidism (PHPT), hyperthyroidism, Paget's disease, myeloma, malignancy, immobility, accelerated osteoporosis, sarcoidosis, and renal tubular acidosis. Besides these conditions, hypercalciuria often recognizes a genetic predisposition manifesting as kidney stones in young patients and with osteoporosis and fragility fractures in postmenopausal women and elder men. Idiopathic hypercalciuria is determined by polymorphic variants/mutations of the calcium sensing receptor (CASR) gene (4-6), mutations of SLC34A1, and SLC34A4 genes $(7,8)$, polymorphic variants of claudins (9), genes involved in calcium reabsorption at the tubular level.

The syndrome of idiopathic hypercalciuria has been described as including low normal serum phosphate, normocalcemia, hypercalciuria, and kidney stones (10). Those individuals with fasting hypercalciuria along with an increased or high/normal parathormone $(\mathrm{PTH})$ are defined as having "renal leak" as the primary mechanism, with the basic abnormality resting within the nephron (10). In clinical practice, distinguishing among intestinal hyperabsorptive hypercalciuria, renal leak hypercalciuria, and idiopathic or fasting hypercalciuria can be difficult (10). Moreover, increased renal calcium excretion is a well-known cause of secondary hyperparathyroidism, where PTH induced bone demineralization by increasing bone resorption. Hypercalciuria is associated with PHPT (11); nonetheless, in a subset of patients with PHPT, surgical resolution of hyperparathyroidism does not associate with urine calcium excretion (UCa) normalization (12). Therefore, it emerges as a spectrum of PTH-related disorders associated with hypercalciuria and with serum calcium levels ranging from normocalcemia to hypercalcemia. It should be also considered that vitamin D and kidney function are important determinants of circulating PTH levels (13). Taken together, all these factors related to hypercalciuria may make it difficult to distinguish PHPT, which may benefit from parathyroidectomy, from non-autonomous PTH-related conditions, where surgery should be avoided as highly probable ineffective in resolving hypercalciuria. In the set of patients with osteoporosis, this diagnostic challenge represents a clinically unmet need, that often increases the number of diagnostic procedures, biochemical tests, and specialist consults proposed to the patients.

Besides the diagnostic challenge, the management and treatment of hypercalciuria are not well defined. Thiazidic diuretics (TZD) are widely used for the management of hypercalciuria among stone-forming patients. TZD stimulates proximal tubular sodium reabsorption as a compensation of their natriuretic action in the distal tubule, resulting in enhanced proximal passive calcium transport (14). Although the effects of different TZD should be relatively similar in terms of prevention of stone recurrence, their potency and side effects may differ. Data concerning the metabolic and bone effects of these agents are limited among recurrent nephrolithiasis patients with hypercalciuria (15). Conceptually, TZD increases tubular calcium reabsorption, thus providing protection against hypercalciuria, and with that may raise serum calcium, suppress PTH secretion, and improve bone metabolism. The additional hypocalciuric effect may be observed with the use of potassiumsparing diuretics (16).

Finally, it should be considered that increased calcium excretion may be due to the increased bone reabsorption and benefit by treatment with anti-resorptive drugs, such as bisphosphonates and denosumab.

In patients affected with osteopenia/osteoporosis, the role of hypercalciuria needs to be elucidated and its management with TZD and/or anti-resorptive drugs needs to be defined.

Focusing on postmenopausal women with reduced bone density, the present study aimed: (1) to describe the different phenotypes associated with hypercalciuria in patients with osteopenia/osteoporosis, pointing on kidney, bone, and biochemical features; and (2) to analyze the efficacy of TZD treatment on reduction of renal calcium excretion, normalization of circulating PTH levels, hypercalcemia development, and on bone mineral density.

\section{MATERIALS AND METHODS}

\section{Data}

Clinical data derived from Osteoregistry, a database collecting real life data from patients referred for osteoporosis management to the third level Endocrinology Service and Rheumatology Unit at IRCCS Istituto Ortopedico Galeazzi in Milan, Italy. All patients gave their informed consent. The study was approved by the 
local ethical committee. All study procedures were performed in compliance with the laws and regulations governing the use of human subjects (Declaration of Helsinki).

\section{Study Design}

This is a retrospective study enrolling 77 osteopenic/osteoporotic women affected with persistent hypercalciuria. Hypercalciuria was defined as UCa $>4.0 \mathrm{mg} / \mathrm{kg}$ body weight $/ 24 \mathrm{~h}$ on at least two determinations on unrestricted diet. Diagnosis of hypercalciuria was established during clinical, biochemical, and hormonal workup for the evaluation of osteoporosis. Exclusion criteria were: male gender; premenopausal women; diagnosis of overt or subclinical hyperthyroidism, endogenous hypercortisolism, Paget's disease, multiple myeloma, malignancy, immobility, sarcoidosis, renal tubular acidosis, corticosteroids, loop diuretics, and hypoalbuminemia.

All patients were investigated by collecting history and anthropometric parameters (weight, height, and body mass index $[\mathrm{BMI}])$. Biochemical profile, such as serum total calcium, phosphate, PTH, creatinine, 25 hydroxyvitamin D (25OHD), total alkaline phosphatase (ALP), and carboxy-terminal collagen crosslinks ( $\beta \mathrm{CTX})$. In patients treated with denosumab, the plasma PTH was assessed at 5 months from the last administration. Plasma ionized calcium was determined in patients with hypercalcemia. The estimated glomerular filtration rate was calculated for each patient according to the Chronic Kidney Disease-Epidemiology Collaboration (CKD-EPI) formula (17). Renal calcium excretions were measured on $24 \mathrm{~h}$ urine collection on at least two distinct occasions in all patients under unrestricted diet. Serum 25OHD levels were measured in all patients at every clinical reassessment and corrected by supplementation with cholecalciferol or calcifediol to maintain serum 25OHD levels above the threshold of $20 \mathrm{ng} / \mathrm{ml}$. Antitransglutaminase autoantibodies were assessed to exclude celiac disease when signs of malabsorption were detected, namely, anemia due to iron insufficiency, or in the presence of other autoimmune manifestations, namely, atrophic gastritis, vitiligo, rheumatoid arthritis, or autoimmune thyroid diseases.

Kidney stones were detected by kidney ultrasound imaging or as a history of symptomatic kidney stones. All patients underwent dual energy X-ray densitometry (DXA) evaluation for the measurement of bone mineral density at lumbar and femur sites (femur neck and hip) by Hologic or Lunar devices. DXA examination was repeated after not less than 18 months. All patients were evaluated for the occurrence of morphometric vertebral fractures by routine dorsal and lumbar X-ray imaging. In addition, clinical fragility fractures were recorded.

\section{Statistical Analysis}

Unsupervised hierarchical clustering analysis by Euclidean wardd2 was performed to identify specific profiles based on mineral metabolic parameters. Principal component analysis (PCA) was calculated to confirm the clustering.

Non-parametric Kruskall-Wallis one-way ANOVA adjusted for multiple testing was performed to analyze the differences among the three mineral metabolic profile-based clusters when variables failed the normality test. Normally distributed variables were analyzed by ordinary one-way ANOVA.

Differences in the biochemical parameters between the two groups were detected by using paired $t$-test or Mann-Withney test for parametric and non-parametric parameters, respectively. Differences in percentages between the two groups were tested by Fisher's exact test. Linear multiple regression considering the treatment with bisphosphonates or denosumab concomitant with TZD as independent variables, and the changes in UCa as the dependent variable was used to test the hypothesis that antiresorptive drugs may contribute to reducing UCa. Parametric data were presented as mean $\pm \mathrm{SD}$, while non-parametric variables were presented as median (range interquartile). Data

TABLE 1 | Clinical and biochemical features of postmenopausal women with low bone mineral density and persistent hypercalciuria.

\begin{tabular}{|c|c|c|}
\hline Parameters & Normal values & \\
\hline N & & 77 \\
\hline Age (years) & & $63.0(57.0-69.0)$ \\
\hline $\mathrm{BMl}\left(\mathrm{kg} / \mathrm{m}^{2}\right)$ & & $23.5(21.9-27.4)$ \\
\hline HRT (\%) & & $7(9.2)$ \\
\hline Active smoke (\%) & & $14(18.0)$ \\
\hline Arterial blood hypertension (\%) & & $7(9.2)$ \\
\hline Familiarity for fragility fractures (\%) & & $15(19.5)$ \\
\hline Cholecalciferol (\%) & & $35(45.5)$ \\
\hline Bisphosphonates (\%) & & $13(16.9)$ \\
\hline \multicolumn{3}{|c|}{ Circulating mineral and bone markers } \\
\hline Serum Ca (mg/dl) & $8.4-10.4$ & $9.6(9.2-10.0)$ \\
\hline Serum P (mg/dl) & $3.0-5.0$ & $3.3(3.0-3.7)$ \\
\hline Plasma PTH (pg/ml) & $15.0-65.0$ & $35.0(42.0-82.1)$ \\
\hline Serum 25OHD (ng/dl) & & $35.0(27.1-41.0)$ \\
\hline Serum total ALP (U/L) & $40.0-120.0$ & $67.0(55.8-92.3)$ \\
\hline Serum $\beta C T X(n g / m l)$ & & $0.510(0.208-0.767)$ \\
\hline \multicolumn{3}{|l|}{ Kidney features } \\
\hline Serum $\operatorname{Cr}(\mathrm{mg} / \mathrm{dl})$ & $<0.80$ & $0.70(0.60-0.78)$ \\
\hline eGFR (ml/min) & $>60.0$ & $93.2(87.9-98.1)$ \\
\hline UCa (mg/24h) & $<400.0$ & $321.0(277.0-405.6)$ \\
\hline UCa (mg/kg/24h) & $<4.0$ & $5.39(4.75-6.70)$ \\
\hline Urine P (mg/kg/24h) & & $815.0(585.0-995.5)$ \\
\hline Kidney stones (\%) & & $25(32.9)$ \\
\hline \multicolumn{3}{|l|}{ Bone features } \\
\hline L1-L4 T-score & $>-2.5 \mathrm{SD}$ & $-2.80(-3.58,-2.03)$ \\
\hline Femur neck T-score & $>-2.5 \mathrm{SD}$ & $-2.20(-2.80,-1.80)$ \\
\hline Total hip T-score & $>-2.5 \mathrm{SD}$ & $-1.87(-2.50,-1.35)$ \\
\hline Osteoporosis at L1-L4 level (\%) & $<-2.5 \mathrm{SD}$ & $46(60.0 \%)$ \\
\hline Osteopenia at L1-L4 level (\%) & $<-1.0,>-2.5 \mathrm{SD}$ & 27 (35.0\%) \\
\hline Osteoporosis at femur neck level (\%) & $<-2.5 \mathrm{SD}$ & $28(36.4 \%)$ \\
\hline Osteopenia at femur neck level (\%) & $<-1.0,>-2.5 \mathrm{SD}$ & $45(58.4 \%)$ \\
\hline Fractures (\%) & & $37(48.7)$ \\
\hline
\end{tabular}

n, number; BMI, body mass index; HRT, hormone replacement therapy; $C a$, total calcium; P, phosphate; PTH, parathormone; $250 H D, 25$ hydroxyvitamin D; ALP, alkaline phosphatase; $\beta C T X, \beta$ carboxy-terminal collagen crosslinks; $\mathrm{Cr}$, creatinine; eGFR, estimated glomerular filtration rate calculated according to the KDIGO CKD-EPI formula (17); UCa, urine calcium excretion. Parameters in the whole series were not normally distributed; they are presented as median (range interquartile) or percentages. 
were analyzed by using Past 3.14 (18) and Prism 6.0. A $P$-value of $<0.05$ was considered statistically significant.

\section{RESULTS}

\section{Clinical and Biochemical Features of Patients With Reduced Bone Mineral Density and Persistent Hypercalciuria}

Clinical and biochemical features of the enrolled postmenopausal women are presented in Table 1. About half of patients were supplemented with cholecalciferol, while $17 \%$ of patients were treated with bisphosphonates. All patients had circulating $25 \mathrm{OHD}$ levels above $20 \mathrm{ng} / \mathrm{ml}$ at the time of basal evaluation.

Kidney features Median (interquartile range) $24 \mathrm{~h}$-UCa was $5.3 \overline{(4.75-6.70)} \mathrm{mg} / \mathrm{kg} / 24 \mathrm{~h}$. Kidney stones were detected anamnestically or by imaging in about one-third of patients, whose UCa was similar to that of patients without kidney stones $[5.33(4.67,7.04)$ vs. $5.40(4.77,6.68) \mathrm{mg} / \mathrm{kg} / 24 \mathrm{~h} ; P=$ $0.814]$. Indeed, patients with kidney stones had lower serum total calcium [9.2 $(9.0,9.8)$ vs. $9.7(9.3,10.0) \mathrm{mg} / \mathrm{dl}, P=0.035]$, while patients with hypercalciuria without kidney stones had significantly lower median lumbar T-score than that detected in patients with kidney stones $[-2.35(-3.1,-1.85)$ vs. $-3.1(-3.7$, $-2.3) ; P=0.018$.

Bone features DXA scan diagnosed osteoporosis (T-score $<-$ $2.5 \mathrm{SD})$ in $60.0 \%$ and osteopenia $(-1.0<$ T-score $<-2.5 \mathrm{SD})$ in $35.0 \%$ of patients at lumbar site and osteoporosis in $36.4 \%$ and osteopenia in $58.4 \%$ of patients at femur neck. At least one fragility fracture occurred in $48.7 \%$ of patients [median 2.0 $(1.0,3.0)]$. UCa did not differ between fractured and unfractured patients $[5.40(4.75,6.89)$ vs. $5.34(4.74,6.43) \mathrm{mg} / \mathrm{kg} / 24 \mathrm{~h} ; P=$ $0.531]$, though fractured patients were older $(65.6 \pm 8.3$ vs. 61.0 \pm 6.3 years; $P=0.007)$, heavier $[25.2(22.4,29.5)$ vs. 22.9 (21.6, 24.8) $\mathrm{kg} / \mathrm{m}^{2} ; P=0.020$ ], and with more severe reduced bone densities [L1-L4 T-score $-3.15(-3.70,-2.50)$ vs. $-2.50(-3.30$, $-1.93), P=0.036$; femur neck T-score $-2.40(-2.98,-1.90)$ vs. $-2.10(-2.70,-1.70), P=0.013$; femur hip T-score -2.20 $(-2.80,-1.50)$ vs. $-1.50(-2.20,-1.10), P=0.003]$.

\section{Mineral Metabolic Parameters-Related Clustering of Hypercalciuric Women With Reduced Bone Mineral Density}

The unsupervised hierarchical cluster analysis by Euclidean wardd 2 considering the three correlated variables serum calcium, phosphate, and PTH, identified two main clusters of patients with hypercalciuria (Figure 1): cluster 1 included 13 patients, cluster 2 included 61 patients, while three patients were excluded from the analysis due to unavailability of serum phosphate determinations. The second order of clusterization identified, in the set of cluster 2 , two subgroups, indicated as cluster $2 \mathrm{~A}$ and cluster $2 \mathrm{~B}$. The main PCA component explaining about $99 \%$ of the variance was determined by PTH values. All patients in cluster 1 presented

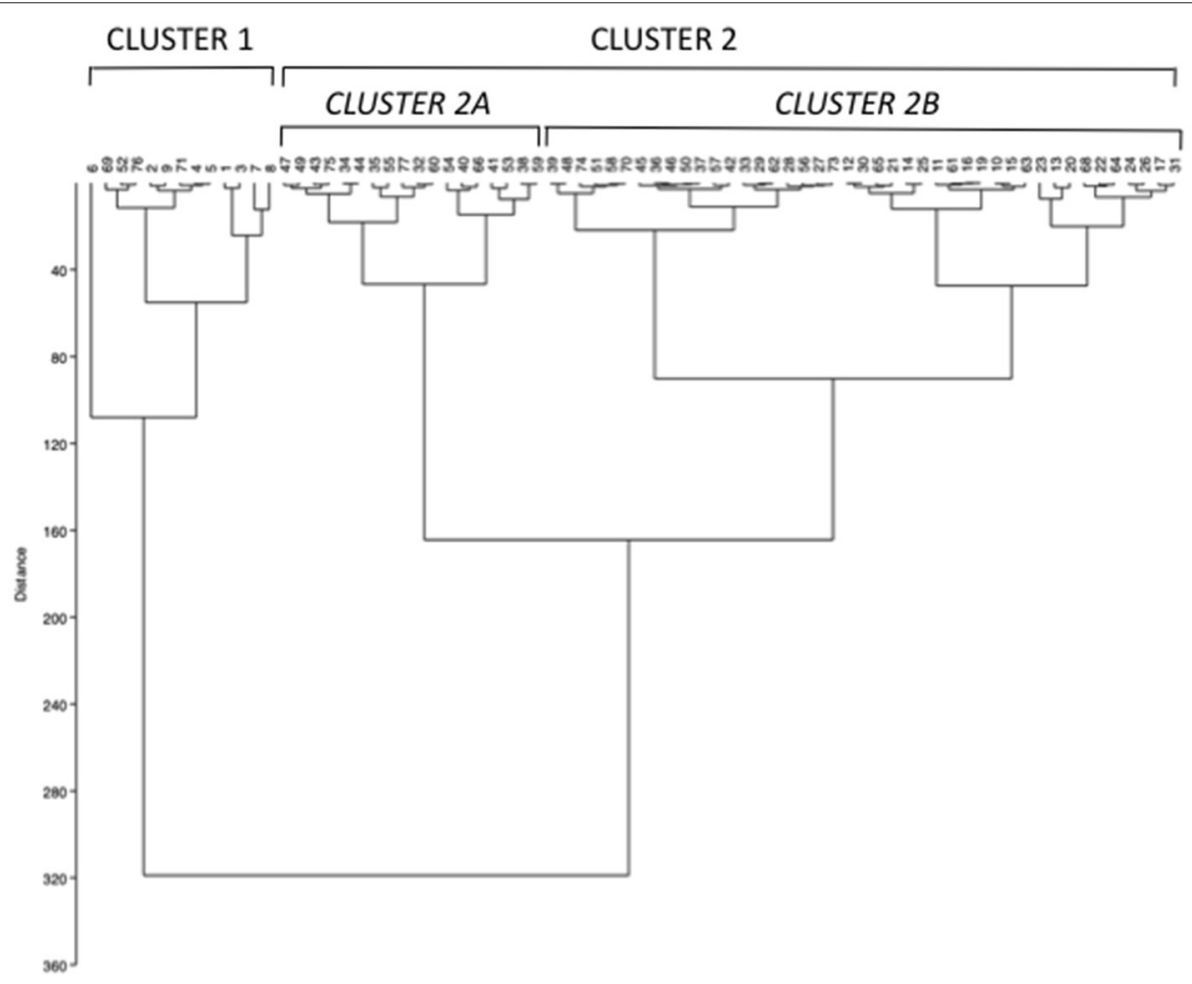

FIGURE 1 | Unsupervised hierarchical cluster analysis by Euclidean wardd2 identifying two main clusters (cluster 1 and 2) based on the mineral metabolic parameters serum calcium, phosphate, and parathormone (PTH) at basal evaluation. A second order of clusterization identified two subgroups in cluster 2 , named cluster $2 \mathrm{~A}$ and $2 \mathrm{~B}$. 
TABLE 2 | Clinical and biochemical features of the clusters of postmenopausal women with reduced bone mineral density and persistent hypercalciuria identified on the base of serum calcium, phosphate and PTH.

\begin{tabular}{|c|c|c|c|c|c|}
\hline \multirow[t]{2}{*}{ Parameters } & \multirow[t]{2}{*}{ N.V. } & \multirow[t]{2}{*}{ Cluster 1} & \multicolumn{2}{|c|}{ Cluster 2} & \multirow[t]{2}{*}{$\mathbf{P}$} \\
\hline & & & Cluster 2A & Cluster 2B & \\
\hline$n$ & & 13 & 18 & 43 & \\
\hline Age (years) & & $63.5 \pm 7.5$ & $64.0 \pm 7.5$ & $62.7 \pm 7.9$ & 0.820 \\
\hline $\mathrm{BMI}\left(\mathrm{kg} / \mathrm{m}^{2}\right)$ & & $24.8(23.4,29.9)$ & $23.5(21.7,29.1)$ & $22.9(21.8,26.7)$ & 0.157 \\
\hline Cholecalciferol (\%) & & 9 (69.2) & $14(77.8)$ & $31(72.1)$ & 0.689 \\
\hline Bisphosphonates (\%) & & $3(23.0)$ & 7 (38.9) & $11(25.6)$ & 0.452 \\
\hline Denosumab (\%) & & $1(7.7)$ & $1(5.5)$ & $1(2.3)$ & 0.414 \\
\hline Serum Ca (mg/dl) & $8.4-10.4$ & $10.0(9.4,10.8)$ & $9.5(9.0,10.3)$ & $9.5(9.2,9.8)^{\star}$ & 0.049 \\
\hline Serum P (mg/dl) & $3.5-5.0$ & $2.8(2.5,3.2)$ & $3.3(2.8,3.6)$ & $3.5(3.2,3.8)^{\star \star}$ & 0.016 \\
\hline Plasma PTH (pg/ml) & $15.0-65.0$ & $121.0(116.0,142.3)$ & $80.5(72.4,91.6)$ & $47.0(37.7,58.0)^{\circ} \S$ & $<0.001$ \\
\hline Serum 250HD (ng/dl) & $>20$ & $36.4(28.2,44.1)$ & $31.4(26.4,40.0)$ & $36.1(27.0,40.0)$ & 0.437 \\
\hline Serum ALP (U/L) & $40-120$ & $84.9 \pm 39.4$ & $65.8 \pm 7.6$ & $70.5 \pm 22.5$ & 0.188 \\
\hline Serum CTX (ng/ml) & & $0.805(0.198,0.837)$ & $0.510(0.066,0.620)$ & $0.463(0.235,0.711)$ & 0.415 \\
\hline Serum Cr (mg/dl) & $<0.9$ & $0.75(0.72,0.77)$ & $0.68(0.60,0.81)$ & $0.68(0.59,0.79)$ & 0.449 \\
\hline eGFR (ml/min) & $>60$ & $94.1(93.2,95.6)$ & $91.1(90.3,98.5)$ & $92.8(86.3,98.9)$ & 0.912 \\
\hline UCa (mg/24h) & $<400$ & $409.2(335.0,487.0)$ & $361.0(274.8,431.2)$ & 303.0 (269.0, 360.0)\# & 0.002 \\
\hline UCa (mg/kg/24h) & $<4.0$ & $5.64(5.30,8.19)$ & $5.8(5.04,6.67)$ & $5.10(4.60,6.69)$ & 0.080 \\
\hline Kidney stones (\%) & & $4(30.8)$ & $4(22.2)$ & $15(34.9)$ & 0.381 \\
\hline L1-L4 T-score & & $-2.80(-3.75,-2.25)$ & $-3.30(-3.63,-2.75)$ & $-2.65(-3.33,-1.90)$ & 0.394 \\
\hline Femur neck T-score & & $-2.00(-2.45,-1.65)$ & $-2.40(-2.95,-1.90)$ & $-2.30(-2.80,-1.79)$ & 0.316 \\
\hline Femur hip T-score & & $-1.65(-2.28,-1.25)$ & $-2.10(-2.80,-1.40)$ & $-1.80(-2.40,-1.15)$ & 0.482 \\
\hline Fractures (\%) & & 5 (38.5) & $10(55.5)$ & $22(51.2)$ & 0.473 \\
\hline
\end{tabular}

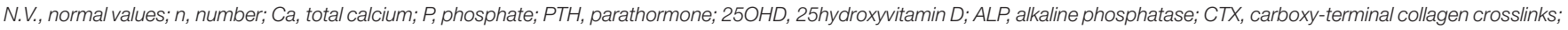

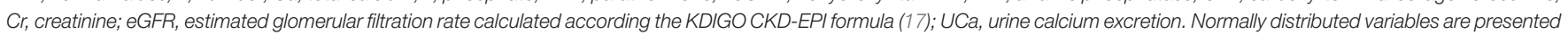

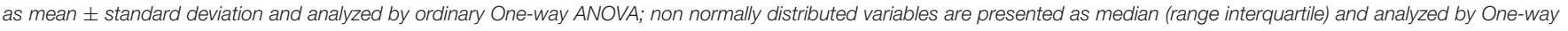
ANOVA Kruskal-Wallis test; $P$-value with statistical significance are indicated in bold.

${ }^{\star} P=0.048$ vs. cluster 1 by Dunn's multiple comparison test.

${ }^{\star \star} P=0.001$ vs. cluster 1 by Dunn's multiple comparison test.

${ }^{\circ} P<0.0001$ vs. cluster 1 by Dunn's multiple comparison test.

$\S_{P}<0.0001$ vs. cluster $2 A$ by Dunn's multiple comparison test.

${ }^{\#} P=0.0015$ vs. cluster 1 by Dunn's multiple comparison test.

the PTH levels higher than the upper limits of the normal range, while all patients in cluster $2 \mathrm{~B}$ presented the $\mathrm{PTH}$ levels within the normal range. Patients in cluster 1 had significantly higher serum total calcium, lower phosphate, and higher PTH levels than patients in cluster $2 \mathrm{~B}$ (Table 2), suggesting a condition of primary hyperparathyroidism due to inappropriate PTH release. Indeed, among patients in cluster 1, three women (23\%) had serum total calcium levels above the upper limit of the normal range $(10.4 \mathrm{mg} / \mathrm{dl})$, of which only one had ionized calcium at the upper limit of the normal range $(1.30 \mathrm{mmol} / \mathrm{L}$; nv. $1.18-1.30$ $\mathrm{mmol} / \mathrm{L})$. Among patients in cluster 2, two women in cluster $2 \mathrm{~A}(11.1 \%)$ and two women in cluster $2 \mathrm{~B}(4.7 \%)$ presented serum calcium levels above the upper normal limit, of which one in cluster $2 \mathrm{~A}$ had concomitantly increased ionized calcium (1.36 mmol/L).

Moreover, cluster 1 patients showed a tendency to present higher median renal calcium excretion than that in patients included in cluster $2 \mathrm{~B}$, though it did not reach the statistical significance. The prevalence of kidney stones was similar in the three clusters. Similarly, lumbar and femur bone mineral densities, as well as fractures prevalence, did not differ among clusters.

Age, BMI, kidney function, circulating bone markers, vitamin D status (evaluated as serum 25OHD levels), cholecalciferol supplementation, and prevalence of ongoing treatment with bisphosphonates or denosumab at time of basal evaluation, were similar among the three clusters (Table 2).

Therefore, cluster analysis suggested a continuum of $\mathrm{PTH}$-dependent alterations in mineral metabolic parameters associated with hypercalciuria in postmenopausal women with reduced bone mineral density.

\section{Effects of Treatment With TZD}

Forty-nine patients were treated with TZD and were reevaluated after $33.4 \pm 19.6$ months. Twenty patients were treated with hydrochlorothiazide (HCT) at a dose ranging 12.5-37.5 $\mathrm{mg} /$ day, while 29 patients were treated with indapamide at a dose ranging $1.50-3.75 \mathrm{mg} /$ day. Any significant difference could be detected between patients treated with HCT and indapamide in the analyzed parameters at both basal and on 
TABLE 3 | Comparison of the effects of indapamide (IND) vs. hydrochlorothiazide (HCT) in postmenopausal women with reduced BMD and persistent hypercalciuria.

\begin{tabular}{|c|c|c|c|c|c|c|}
\hline \multirow[b]{2}{*}{ Parameters } & \multicolumn{2}{|c|}{ BASAL } & \multirow[b]{2}{*}{$\mathbf{P}$} & \multicolumn{2}{|c|}{ TREATED } & \multirow[b]{2}{*}{$\boldsymbol{P}$} \\
\hline & IND & HCT & & IND & HCT & \\
\hline$n$ & 29 & 20 & & 29 & 20 & \\
\hline Follow up (months) & $30.0(20.5,38.0)$ & $36.0(19.0,48.0)$ & 0.259 & & & \\
\hline $\mathrm{BMI}\left(\mathrm{kg} / \mathrm{m}^{2}\right)$ & $25.0 \pm 4.6$ & $26.7 \pm 5.2$ & 0.231 & & & \\
\hline Age (years) & $61.8 \pm 7.4$ & $62.6 \pm 6.4$ & 0.682 & & & \\
\hline Serum Ca (mg/dl) & $9.6 \pm 0.6$ & $9.7 \pm 0.6$ & 0.513 & $9.7(9.3,10.0)$ & $9.6(9.3,10.1)$ & 0.820 \\
\hline Serum P (mg/dl) & $3.3(3.1,3.6)$ & $3.4(2.8,3.8)$ & 0.896 & $3.2(2.8,3.4)$ & $3.5(3.1,3.7)$ & 0.087 \\
\hline Plasma PTH (pg/ml) & $62.0(54.0,88.4)$ & $53.1(43.3,93.7)$ & 0.371 & $54.4(48.2,72.1)$ & $48.5(36.0,80.0)$ & 0.548 \\
\hline 25OHD (ng/dl) & $37.0 \pm 12.3$ & $35.7 \pm 15.2$ & 0.737 & $41.8(33.0,47.7)$ & $42.3(35.0,63.0)$ & 0.569 \\
\hline UCa (mg/kg/24 h) & $6.0 \pm 1.7$ & $5.6 \pm 1.1$ & 0.355 & $3.8 \pm 1.4$ & $3.2 \pm 1.1$ & 0.139 \\
\hline$\Delta \mathrm{UCa}(\mathrm{mg} / \mathrm{kg} / 24 \mathrm{~h})$ & & & & $-0.35(-0.49,-0.26)$ & $-0.45(-0.52,-0.27)$ & 0.272 \\
\hline UCa <4 mg/kg/24h (\%) & & & & $19(65.5)$ & $14(70)$ & 0.768 \\
\hline eGFR $\left(\mathrm{ml} / \mathrm{min} / 1.73 \mathrm{~m}^{2}\right)$ & $93.8(90.8,98.4)$ & $92.5(88.8,98.9)$ & 0.865 & $90.7(86.1,96.8)$ & $91.7(87.6,96.2)$ & 0.838 \\
\hline L1-L4 T-score & $-2.83 \pm 1.05$ & $-2.76 \pm 1.13$ & 0.803 & $-2.68 \pm 1.00$ & $-2.33 \pm 1.15$ & 0.327 \\
\hline Femur neck T-score & $-2.28 \pm 0.66$ & $-2.26 \pm 0.65$ & 0.919 & $-2.27 \pm 0.81$ & $-2.08 \pm 0.75$ & 0.448 \\
\hline Femur hip T-score & $-1.90 \pm 0.78$ & $-1.88 \pm 0.96$ & 0.940 & $-1.85 \pm 0.73$ & $-1.73 \pm 0.73$ & 0.606 \\
\hline
\end{tabular}

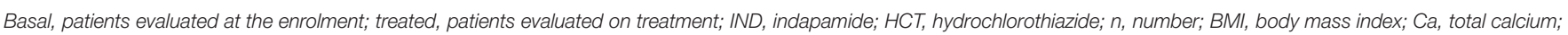

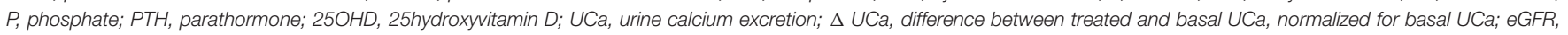

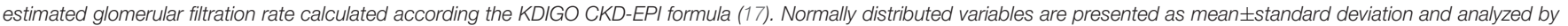
T-test of Student; non normally distributed variables are presented as median (range interquartile) and analyzed by Mann-Whitney test.

treatment evaluations. In particular, patients treated with HCT or indapamide showed similar reductions in renal calcium excretion (Table 3). Therefore, patients treated with HCT or indapamide were analyzed as a unique series. At the time of enrollment, $71.4 \%$ of patients were supplemented with cholecalciferol, while about a quarter was treated with bisphosphonates for a period not longer than 12 months. At the time of re-evaluation on TZD treatment, almost all patients were supplemented with cholecalciferol, all patients treated with bisphosphonates maintained their therapy, while about one-third started treatment with denosumab.

\section{Effects of TZD on UCa and PTH Levels}

Patients on TZD treatment showed a significant reduction of renal calcium excretion with a mean decrease of $39 \%$ of basal levels (Figure 2A), determining normalization of the urine calcium levels in 31 (63.3\%) patients. Moreover, TZD treatment reduced median circulating PTH levels (Figure 2B). Considering the concomitant treatment with bisphosphonates or denosumab as independent variables, linear multiple regression showed that the TZD-induced changes in $\mathrm{UCa}$ is significantly related to treatment with denosumab (coefficient $-0.147, P=0.016$ ).

The same pattern of response was observed considering the subset of patients treated with TZD in absence of concomitant bone anti-resorptive therapy (both bisphosphonates and denosumab; $n=19$ ) (Table 4). In particular, TZD treatment determined significant reductions of $\mathrm{UCa}$ and $\mathrm{PTH}$ levels. At variance, a mild increase $(0.3 \mathrm{mg} / \mathrm{dl})$ of serum total calcium could be detected. Of note, the entity of the TZD-induced PTH reduction was significantly higher in patients not concomitantly treated with anti-resorptive agents (bisphosphonates, denosumab, $\Delta$ basal PTH-post TZD PTH levels, $20.3 \pm 24.6$ vs. $6.2 \pm 22.4 \mathrm{pg} / \mathrm{ml}, P=0.045$ by $T$ Student's test).

\section{Effects of TZD on Mineral Metabolic Profile}

We analyzed the effect of the TZD treatment in the three mineral metabolic profiles identified by cluster analysis at basal evaluation (Table 5). TZD treatment induced an efficient reduction of the $\mathrm{UCa}$ independently from the cluster in which patients were included. The TZD-induced reduction in UCa was associated with the reduced PTH levels in patients included in clusters 1 and $2 \mathrm{~A}$, while any change was observed in patients included in cluster 2B, who had circulating PTH levels in the normal range. TZD treatment did not induce any significant change in serum calcium and phosphate levels in patients of the three clusters.

\section{Effects of TZD on Bone Mineral Density}

Bone mineral densities at lumbar and femur sites did not vary significantly after TZD treatment in patients without concomitant anti-resorptive drugs (Table 4).

Of note, considering the subset of women concomitantly treated with TZD and anti-resorptives (bisphosphonates or denosumab, $n=25$ ), we found that patients gaining normalization of the UCa (UCa $<4 \mathrm{mg} / \mathrm{kg} / 24 \mathrm{~h} ; n=17$ ) experienced significant increases in bone mineral densities at lumbar (L1-L4 T-score $-3.12 \pm 1.11$ vs. $-2.85 \pm 1.09, P=$ 0.032 ) and femur neck (femur neck T-score $-2.43 \pm 0.62$ vs. $-2.23 \pm 0.75, P=0.077)$. By contrast, patients with persistent hypercalciuria on TZD treatment did not show significant changes in bone mineral densities (L1-L4 T-score $-2.99 \pm 1.04$ vs. $-2.95 \pm 1.24, P=0.903$; femur neck T-score $-2.31 \pm 0.77$ vs. $-2.61 \pm 0.70, P=0.319)$. 


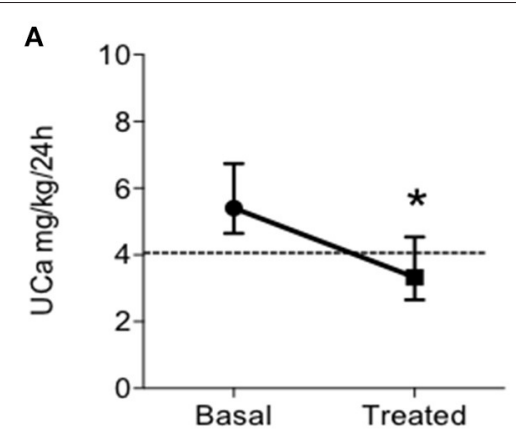

B

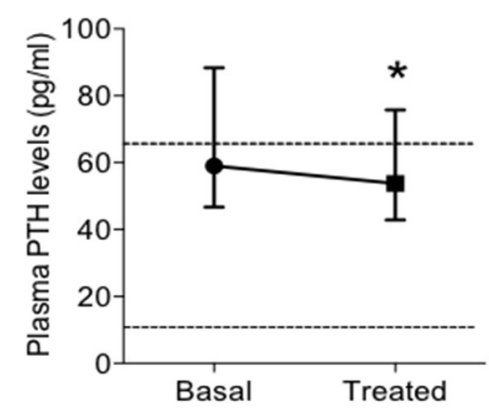

FIGURE 2 | Effects of treatment with thiazidic diuretics (TZD) on urine calcium excretion (UCa) (A) and plasma PTH levels (B) in postmenopausal women with reduced bone mineral density and persistent hypercalciuria $(n=49)$. UCa, urine calcium excretion; and PTH, parathormone. ${ }^{*}, P=0.005$.

\section{DISCUSSION}

We retrospectively investigated a consistent series of postmenopausal women with osteopenia/osteoporosis and persistent hypercalciuria in the presence of sufficient $25 \mathrm{OHD}$ levels (19). Kidney stones occurred in one-third of patients, whose UCa did not differ from that in women who did not experience kidney stones, suggesting that the screening for hypercalciuria should be performed also in patients with no history of recurrent kidney stones. Lithogenesis is associated with individual and environmental factors. Individual factors include metabolic disturbance, inheritance, urinary tract infection, and obstruction (20). Of note, in patients with primary hyperparathyroidism, relative hyperoxaluria (21), hypomagnesuria (22) as well as polymorphic variants of the gene encoding the calcium sensing receptor (23) have been identified as factors significantly associated with kidney stones.

Unsupervised hierarchical clustering analysis identified a spectrum of mineral metabolic profiles in hypercalciuric women with reduced bone mineral density, similar to what was reported in patients with nephrolithiasis (10). Mineral metabolic profiles range from features resembling PHPT with reduced phosphate levels (cluster 1) to hyperparathyroidism with normal phosphate levels (cluster 2A) to profile with all mineral parameters within the normal range (cluster $2 \mathrm{~B}$ ). $\mathrm{PTH}$ was the main determinant of the mineral metabolic
TABLE 4 | Effects of thiazidic diuretics (TZD) treatment on biochemical parameters in postmenopausal women with reduced BMD and persistent hypercalciuria not treated with anti-resorptives.

\begin{tabular}{lcccc}
\hline Parameters & BASAL & TZD & $\begin{array}{c}\boldsymbol{\Delta} \text { Basal- } \\
\text { Treated }\end{array}$ & $\boldsymbol{P}$ \\
& & & & \\
$\mathrm{N}$ & 19 & 19 & -0.3 & $\mathbf{0 . 0 3 2}$ \\
Serum Ca (mg/dl) & $9.5 \pm 0.6$ & $9.8 \pm 0.5$ & 0.01 & 0.967 \\
Serum P (mg/dl) & $3.29 \pm 0.52$ & $3.29 \pm 0.52$ & 20.3 & $\mathbf{0 . 0 0 2}$ \\
Plasma PTH (pg/ml) & $78.3 \pm 38.9$ & $57.9 \pm 25.8$ & -4.94 & 0.290 \\
25OHD (ng/dl) & $37.7 \pm 16.2$ & $42.7 \pm 9.4$ & 1.94 & $<\mathbf{0 . 0 0 1}$ \\
UCa (mg/kg/24h) & $5.84 \pm 1.62$ & $3.90 \pm 1.14$ & 1.5 & 0.209 \\
eGFR (ml/min/1.73m²) & $94.1 \pm 5.6$ & $92.6 \pm 6.3$ & -0.05 & 0.699 \\
L1-L4 T-score & $-2.92 \pm 1.00$ & $-1.97 \pm 0.95$ & -0.05 & 0.655 \\
Femur neck T-score & $-1.93 \pm 0.65$ & $-1.87 \pm 0.77$ & -0.03 & 0.774 \\
Femur hip T-score & $-1.46 \pm 0.66$ & $-1.43 \pm 0.58$ & -1.95
\end{tabular}

Basal, patients evaluated at the enrolment; TZD, patients evaluated on TZD treatment; $\triangle$ Basal-treated, the difference between basal and treated values; $n$, number; $C a$, total calcium; P, phosphate; PTH, parathormone; 25OHD, 25hydroxyvitamin D; UCa, urine calcium excretion; eGFR, estimated glomerular filtration rate calculated according to the $K D I G O C K D$-EPI formula (17). Normally distributed variables are presented as mean $\pm S D$ and analyzed by T-test of Student's test; non-normally distributed variables are presented as median (range interquartile) and analyzed by Mann-Whitney test; P-value with statistical significance is indicated in bold.

profile associated with hypercalciuria and reduced bone mineral density. The detection of low phosphate levels in the presence of hyperparathyroidism is consistent with the phosphaturic action of PTH at kidney level; the concomitant tendency to higher calcium levels and higher renal calcium excretion were suggestive of a certain degree of inappropriate PTH secretion, identifying a condition distinct from that detected in cluster $2 \mathrm{~A}$ patients, were increased $\mathrm{PTH}$ levels were likely secondary to hypercalciuria. Indeed, it should be noted that the mineral metabolic profile in patients included in clusters 1 and $2 \mathrm{~A}$ showed considerable overlap. Of note, in the present series of hypercalciuric postmenopausal osteoporotic women, hypercalcemic PHPT is rare, suggesting that diagnosis of normocalcemic or hypercalcemic PHPT needs to be carefully evaluated as well as an indication to parathyroid surgery. Unfortunately, any biochemical or clinical parameters correlated with the mineral metabolic profile.

We further investigated the effect of TZD treatment in this series of patients. TZD effectively reduced renal calcium excretion with a mean decrease of $39 \%$ of basal levels and determined a normalization of UCa in about two-thirds of patients. This pattern of response to TZD treatment was confirmed in the subgroup of patients free from anti-resorptive drugs. Of note, in the whole series of TZD treated patients, concomitant treatment with denosumab contribute to further reduction of UCa, while bisphosphonates were neutral. Moreover, TZD treatment reduces the PTH levels in patients with hyperparathyroidism, suggesting that the increased PTH levels are at least in part secondary to hypercalciuria. This is in line with what was reported by Eisner et al. (24), who proposed the "thiazide challenge" test to discriminate between primary and secondary hyperparathyroidism in patients with recurrent kidney stones based on normalization 
TABLE 5 | Effects of thiazidic diuretics (TZD) treatment on clinical and metabolic parameters in 49 postmenopausal women treated with TZD.

\begin{tabular}{|c|c|c|c|c|c|c|c|c|c|}
\hline \multirow[b]{3}{*}{ Parameters } & \multicolumn{3}{|c|}{ CLUSTER 1} & \multicolumn{6}{|c|}{ CLUSTER 2} \\
\hline & \multirow[b]{2}{*}{ Basal } & \multirow[b]{2}{*}{ TZD } & \multirow[b]{2}{*}{$\mathbf{P}$} & \multicolumn{3}{|c|}{ CLUSTER 2A } & \multicolumn{3}{|c|}{ CLUSTER $2 B$} \\
\hline & & & & Basal & TZD & $\mathbf{P}$ & Basal & TZD & $\mathbf{P}$ \\
\hline$n$ & 7 & 7 & & 13 & 13 & & 29 & 29 & \\
\hline Follow up (months) & & $48.0 \pm 33.5$ & & & $25.1 \pm 15.1^{*}$ & & & $31.8 \pm 15.3$ & \\
\hline Age (years) & $63.1 \pm 6.9$ & & & $61.7 \pm 6.6$ & & & $61.6 \pm 7.0$ & & \\
\hline BMI $\left(\mathrm{kg} / \mathrm{m}^{2}\right)$ & $28.3 \pm 5.0$ & & & $26.7 \pm 5.3$ & & & $24.4 \pm 4.5$ & & 0.107 \\
\hline Cholecalciferol (\%) & $5(71)$ & $6(86)$ & 0.633 & $9(69)$ & $13(100)$ & 0.096 & $20(69)$ & $29(100)$ & 0.002 \\
\hline Bisphosphonates (\%) & $2(29)$ & $0(0)$ & 1.000 & $5(38)$ & $4(31)$ & 1.000 & $6(21)$ & $9(31)$ & 0.550 \\
\hline Denosumab (\%) & $0(0)$ & $3(43)$ & 0.192 & $0(0)$ & $4(31)$ & 0.096 & $1(5)$ & $7(24)$ & 0.052 \\
\hline Serum Ca (mg/dl) & $9.8 \pm 0.7$ & $\begin{array}{c}10.1 \\
(9.3,10.1)\end{array}$ & 0.597 & $9.6 \pm 0.7$ & $\begin{array}{c}9.7 \\
(9.5,10.1)\end{array}$ & 0.105 & $9.7 \pm 0.5$ & $\begin{array}{c}9.5 \\
(9.1,9.9)\end{array}$ & 0.358 \\
\hline Serum P (mg/dl) & $2.8(2.5,3.8)$ & $2.7 \pm 0.7$ & 0.468 & $3.4(2.7,3.8)$ & $3.2 \pm 0.6$ & 0.387 & $\begin{array}{c}3.4 \\
(3.1,3.7)\end{array}$ & $3.4 \pm 0.4 \S$ & 0.266 \\
\hline Plasma PTH (pg/ml) & $\begin{array}{c}145.6 \pm \\
38.9^{\star \star}\end{array}$ & $106.9 \pm 63.3$ & 0.017 & $83.3 \pm 11.2^{\star \star}$ & $64.7 \pm 23.4^{\circ}$ & 0.009 & $47.7 \pm 13.3^{\star *}$ & $47.2 \pm 16.8^{\circ}$ & 0.745 \\
\hline Serum 25OHD (ng/ml) & $39.9 \pm 14.0$ & $\begin{array}{c}46.0 \\
(28.8,63.2)\end{array}$ & 0.394 & $31.1 \pm 9.6$ & $\begin{array}{c}41.8(30.1 \\
51.1)\end{array}$ & 0.036 & $37.7 \pm 14.6$ & $\begin{array}{c}41.5 \\
(35.1,48.4)\end{array}$ & 0.018 \\
\hline eGFR $\left(\mathrm{ml} / \mathrm{min} / 1.73 \mathrm{~m}^{2}\right)$ & $\begin{array}{c}94.7 \\
(93.1,95.7)\end{array}$ & & & $\begin{array}{c}94.7 \\
(90.5,98.8)\end{array}$ & & & $\begin{array}{c}93.6 \\
(88.9,99.0)\end{array}$ & & \\
\hline UCa (mg/24h) & $\begin{array}{c}422.2 \\
(384.0,504.0)\end{array}$ & $253.5 \pm 100.3$ & 0.003 & $\begin{array}{c}372.0 \\
(312.2,454.5)\end{array}$ & $\begin{array}{c}263.0 \pm \\
83.8\end{array}$ & $<0.001$ & $\begin{array}{c}313.7 \\
(270.5,371.0) \#\end{array}$ & $204.7 \pm 78.3$ & $<0.001$ \\
\hline UCa (mg/kg/24 h) & $6.42 \pm 1.94$ & $3.72 \pm 1.82$ & 0.005 & $5.79 \pm 1.01$ & $4.01 \pm 1.11$ & $<0.001$ & $5.74 \pm 1.58$ & $3.40 \pm 1.27$ & $<0.001$ \\
\hline Kidney stones (\%) & $4(57.1)$ & & & $3(23.0)$ & & & $13(44.8)$ & & \\
\hline
\end{tabular}

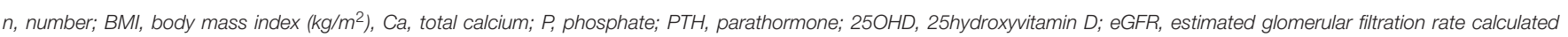

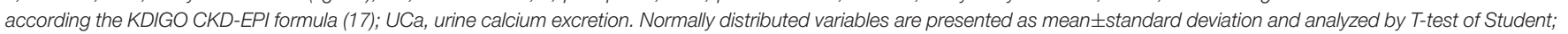
non normally distributed variables are presented as median (range interquartile) and analyzed by Mann-Whitney test; P-value with statistical significance are indicated in bold.

${ }^{*} P=0.035$ vs. Cluster 1 at basal evaluation by ordinary One-way ANOVA.

${ }^{* \star} P<0.05$ among Cluster 1 , Cluster $A$ and Cluster $B$ by ordinary One-way ANOVA.

$\S P=0.013$ vs. Cluster 1 at on treatment evaluation by ordinary One-way ANOVA.

${ }^{\circ} P<0.01$ vs. Cluster 1 at on treatment evaluation by ordinary One-way ANOVA.

${ }^{\#} P=0.016$ vs. Cluster 1 at basal evaluation by Dunn's multiple comparison test.

of PTH levels after administration of hydrochlorotiazide $25 \mathrm{mg}$ two times a day for 2 weeks. Indeed, patients in the present series were treated with lower doses of TZD. Considering the whole series, we observed that antiresorptive drugs may blunt the TZD-induced reduction of the PTH levels.

The follow-up of TZD treated patients, though of variable duration in every single patient, showed that bone mineral densities at both lumbar and femur sites were unaffected by TZD treatment alone. Of interest, patients with TZD-induced normalization of UCa experienced a significant increase of bone mineral densities when concomitantly treated with antiresorptive drugs, while such positive effect could not be detected in patients with persistent hypercalciuria. Though coming from a series of limited sample size, data support the utility to treat hypercalciuria with TZD in hypercalciuric postmenopausal women with osteopenia/osteoporosis in addition to antiresorptives when the risk of fracture is increased. Of note, present data suggest that denosumab may contribute to reducing $\mathrm{UCa}$, while bisphosphonates have no effect.
Admittedly, the retrospective design of the study represents a limitation as well as the heterogeneity of the hydrochlorothiazide and indapamide doses and the anti-resorptive treatment. Moreover, the real-life setting prevented properly assessing the dietary calcium intake. Nonetheless, we provide data from the real-life setting about clinical and biochemical features of hypercalciuria and the efficacy of treatment with TZD and anti-resorptives, which highlighted the clinical role of hypercalciuria in the management of patients with osteoporosis and prompted the need for randomized controlled studies.

In conclusion, in hypercalciuric postmenopausal women with reduced bone mineral density, alterations of the mineral metabolic profile may represent a diagnostic challenge in distinguishing PHPT from secondary hyperparathyroidism. In a real-life clinical setting treatment with TZD is effective in reducing and normalizing UCa and PTH levels with any significant difference between indapamide and hydrochlorotiazide. Of note, patients with normalized urine calcium excretion by TZD treatment experienced 
significant increases in bone mineral densities when treated with anti-resorptives, bisphosphonates, or denosumab.

\section{DATA AVAILABILITY STATEMENT}

The datasets presented in this study can be found in online repositories. The names of the repository/repositories and accession number(s) can be found at: https://doi.org/10.5281/ zenodo. 5516995 .

\section{ETHICS STATEMENT}

The studies involving human participants were reviewed and approved by IRCCS Ospedale San Raffaele Milano. The

\section{REFERENCES}

1. Letavernier E, Daudon M. Vitamin D, hypercalciuria and kidney stones. Nutrients. (2018) 10:366. doi: 10.3390/nu100 30366

2. Saponaro F, Cetani F, Mazoni L, Apicella M, Di Giulio M, Carlucci F, et al. Hypercalciuria: its value as a predictive risk factor for nephrolithiasis in asymptomatic primary hyperparathyroidism? J Endocrinol Invest. (2020) 43:677-82. doi: 10.1007/s40618-019-0 1162-y

3. Eller-Vainicher C, Cairoli E, Zhukouskaya VV, Morelli V, Palmieri S, Scillitani A, et al. Prevalence of subclinical contributors to low bone mineral density and/or fragility fracture. Eur J Endocrinol. (2013) 169:22537. doi: 10.1530/EJE-13-0102

4. Arcidiacono T, Mingione A, Macrina L, Pivari F, Soldati L, Vezzoli G. Idiopathic calcium nephrolithiasis: a review of pathogenic mechanisms in the light of genetic studies. Am J Nephrol. (2014) 40:499-506. doi: 10.1159/0003 69833

5. Mingione A, Verdelli C, Terranegra A, Soldati L, Corbetta S. Molecular and clinical aspects of the target therapy with the calcimimetic cinacalcet in the treatment of parathyroid tumors. Curr Cancer Drug Targets. (2015) 15:563-74. doi: 10.2174/15680096156661506021 43041

6. Vezzoli G, Macrina L, Magni G, Arcidiacono T. Calciumsensing receptor: evidence and hypothesis for its role in nephrolithiasis. Urolithiasis. (2019) 47:23-33. doi: 10.1007/s00240-0181096-0

7. Bergwitz C, Miyamoto KI. Hereditary hypophosphatemic rickets with hypercalciuria: pathophysiology, clinical presentation, diagnosis and therapy. Pflugers Arch. (2019) 471:149-63. doi: 10.1007/s00424-0182184-2

8. Gordon RJ, Li D, Doyle D, Zaritsky J, Levine MA. Digenic heterozygous mutations in SLC34A3 and SLC34A1 cause dominant hypophosphatemic rickets with hypercalciuria. J Clin Endocrinol Metab. (2020) 105:2392-400. doi: 10.1210/clinem/dg aa217

9. Hou J. Claudins and mineral metabolism. Curr Opin Nephrol Hypertens. (2016) 25:308-13. doi: 10.1097/MNH.00000000000 00239

10. Ryan LE, Ing SW. Idiopatic hypercalciuria and bone health. Curr Osteoporos Rep. (2012) 10:286-95. doi: 10.1007/s11914-0120120-5

11. Minisola S, Gianotti L, Bhadada S, Silverberg SJ. Classical complications of primary hyperparathyroidism. Best Pract Res Clin Endocrinol Metab. (2018) 32:791-803. doi: 10.1016/j.beem.2018. 09.001 patients/participants provided their written informed consent to participate in this study.

\section{AUTHOR CONTRIBUTIONS}

FN collected and analyzed data. GD, GG, and ML managed patients. SC conceived the study, reviewed the statistical analysis, and wrote the manuscript. All authors reviewed, discussed, and approved the final version of the manuscript.

\section{FUNDING}

The study was supported by the GSD Foundation 5x1000 fund to Osteoregistry and the Italian Ministry of Health.

12. Palmieri S, Eller-Vainicher C, Cairoli E, Morelli V, Zhukouskaya VV, Verga U, et al. Hypercalciuria may persist after successful parathyroid surgery and it is associated with parathyroid hyperplasia. J Clin Endocrinol Metab. (2015) 100:2734-42. doi: 10.1210/jc. 2014-4548

13. Marcocci C, Brandi ML, Scillitani A, Corbetta S, Faggiano A, Gianotti L, et al. Italian society of endocrinology consensus statement: definition, evaluation and management of patients with mild primary hyperparathyroidism. J Endocrinol Invest. (2015) 38:577-93. doi: 10.1007/s40618-0150261-3

14. Nijenhuis T, Vallon V, van der Kemp AW, Loffing J, Hoenderop JG, Bindels RJ. Enhanced passive $\mathrm{Ca} 2+$ reabsorption and reduced $\mathrm{Mg} 2+$ channel abundance explains thiazide-induced hypocalciuria and hypomagnesemia. J Clin Invest. (2005) 115:1651-8. doi: 10.1172/JCI 24134

15. Li DF, Gao YL, Liu HC, Huang XC, Zhu RF, Zhu CT. Use of thiazide diuretics for the prevention of recurrent kidney calculi: a systematic review and meta-analysis. J Transl Med. (2020) 18:106. doi: 10.1186/s12967-020-0 2270-7

16. Alon US. The effects of diuretics on mineral and bone metabolism. Pediatr Endocrinol Rev. (2018) 15:291-7. doi: 10.17458/per.vol 15.2018

17. Levey AS, Inker LA, Coresh J. GFR estimation: from physiology to public health. Am J Kidney Dis. (2014) 63:820-34. doi: 10.1053/j.ajkd.2013. 12.006

18. Hammer $\varnothing$, Harper DAT, Ryan PD. PAST: Paleontological Statistics Software Package For Education. Purcellville: Coquina Press. (2001).

19. Cesareo R, Attanasio R, Caputo M, Castello R, Chiodini I, Falchetti A, et al. Italian Association of Clinical Endocrinologists (AME) and Italian Chapter of the American Association of Clinical Endocrinologists (AACE) position statement: clinical management of vitamin D deficiency in adults. Nutrients. (2018) 10:546. doi: 10.3390/nu100 50546

20. Moriyama MT. Diet therapy and life guidance to prevent calcium stones. Clin Calcium. (2011) 21:1522-9.

21. Corbetta S, Baccarelli A, Aroldi A, Vicentini L, Fogazzi GB, Eller-Vainicher C, et al. Risk factors associated to kidney stones in primary hyperparathyroidism. $J$ Endocrinol Invest. (2005) 28:122-8. doi: 10.1007/BF033 45354

22. Verdelli C, Corbetta S. MECHANISMS IN ENDOCRINOLOGY: Kidney involvement in patients with primary hyperparathyroidism: an update on clinical and molecular aspects. Eur J Endocrinol. (2017) 176:R39-52. doi: 10.1530/EJE16-0430

23. Saponaro F, Marcocci C, Apicella M, Mazoni L, Borsari S, Pardi E, et al. Hypomagnesuria is associated with nephrolithiasis in patients with 
asymptomatic primary hyperparathyroidism. J Clin Endocrinol Metab. (2020) 105:dgaa233. doi: 10.1210/clinem/dgaa233

24. Eisner BH, Ahn J, Stoller ML. Differentiating primary from secondary hyperparathyroidism in stone patients: the "thiazide challenge". J Endourol. (2009) 23:191-2. doi: 10.1089/end.2008.0567

Conflict of Interest: The authors declare that the research was conducted in the absence of any commercial or financial relationships that could be construed as a potential conflict of interest.

Publisher's Note: All claims expressed in this article are solely those of the authors and do not necessarily represent those of their affiliated organizations, or those of the publisher, the editors and the reviewers Any product that may be evaluated in this article, or claim that may be made by its manufacturer, is not guaranteed or endorsed by the publisher.

Copyright $\odot 2021$ Nicoli, Dito, Guabello, Longhi and Corbetta. This is an open-access article distributed under the terms of the Creative Commons Attribution License (CC $B Y)$. The use, distribution or reproduction in other forums is permitted, provided the original author(s) and the copyright owner(s) are credited and that the original publication in this journal is cited, in accordance with accepted academic practice. No use, distribution or reproduction is permitted which does not comply with these terms. 